\title{
A review of the management of oral drug overdose in the Accident and Emergency Department of the Royal Brisbane Hospital
}

D. W. HODGKINSON, L. B. JELLETT \& R. H. ASHB Yọ

The Accident and Emergency Department, Royal Brisbane Hospital, Herston Road, Brisbane, Australia

SUMMARY

Two-hundred and eighty-nine patients who made a total of 323 presentations to the Royal Brisbane Hospital Accident and Emergency Department with a known or suspected oral drug overdose were reviewed. The majority of patients $(76 \%)$ could be managed in a $24 \mathrm{~h}$ Accident and Emergency observation unit. Activategd charcoal given orally or via a nasogastric tube was the recommended method of preventing further absorption of an ingested drug. The use of syrup of ipecac wås not encouraged and orogastric lavage was used in only specific situations. The morbidity and mortality of these patients when compared with other studies, was $\stackrel{\circ}{\circ}$ not adversely affected by this protocol which dramatically reduced the indications $\overrightarrow{0}$ for the use of orogastric lavage and syrup of ipecac.

\section{INTRODUCTION}

The Royal Brisbane Hospital Accident and Emergency Department is a large adult $\frac{\hat{\delta}}{3}$ Emergency Department. It serves a population of 600000 and attends to 85000 new $_{\circ}$ cases per year. It has adopted a conservative management policy with regard to gastric emptying procedures and recommends orogastric lavage only in specific을. situations (Fig. 1). Activated charcoal is recommended routinely for the treatment of oral drug overdose. In the U.K. at the present time the preferred methods of preventing further absorption of an orally ingested drug are orogastric lavage or: induction of emesis with syrup of ipecac. Orogastric lavage is an unpleasant ${ }_{\sigma}^{\omega}$ procedure, not without risk (Thompson et al., 1987) and the basis for its routine

Correspondence: Dr D. W. Hodgkinson, Hope Hospital, Eccles Old Road, Salford, Manchester M6 8HD, U.K. T 


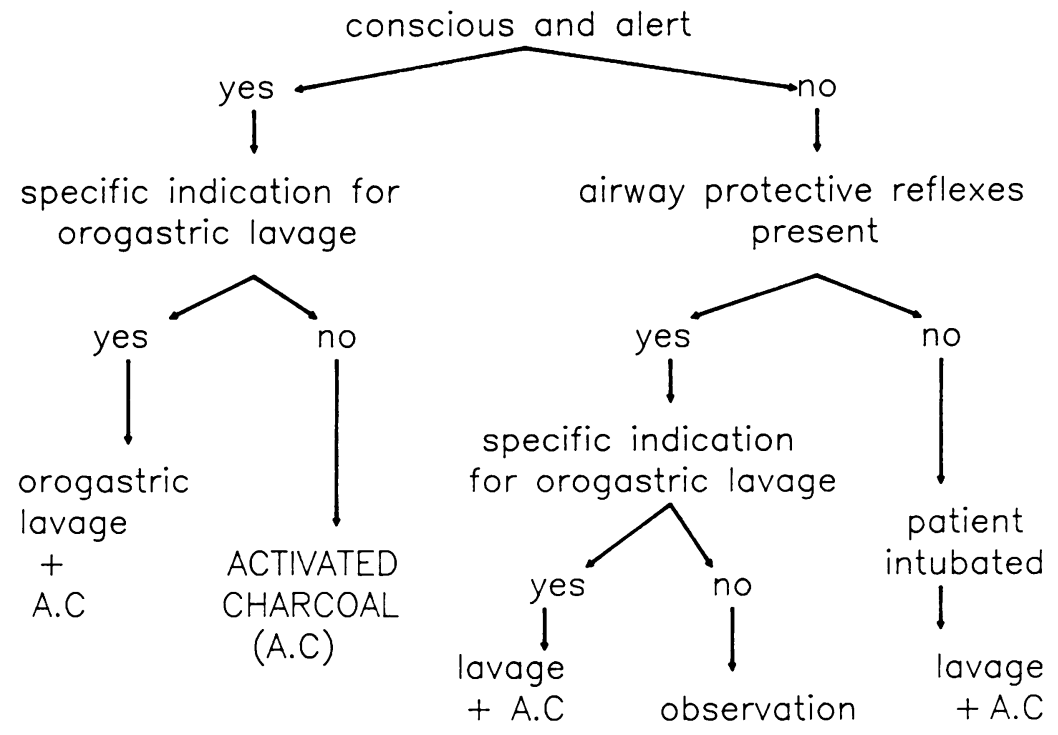

Fig. 1 Algorithm: management of the overdose patient.

A specific indication for orogastric lavage was judged to be a potentially serious ingestion any of the following drugs: salicylate, paracetamol, paraquat, iron, quinine, digoxin, b-blocker tricyclic antidepressant, colchicine. The potential seriousness relates to the time since ingestion and the quantity taken.

use rests more on the occasional recovery of large quantities of drug rather than verification of its routine efficiency by studies in man (Melman \& Morelli, 1978; Wheeler-Usher, 1986).

Syrup of ipecac has been used successfully in children. In adults it has not been shown to be superior to activated charcoal in reducing absorption of an orally ingested substance. There is the added problem of a sedative drug being the most common drug taken in overdose in adults and aspiration of vomit the most common cause of morbidity and mortality in these patients (Nielson \& Henry, 1984). This study reviewed the patients presenting to the Accident and Emergency Department of the Royal Brisbane Hospital with a known or suspected oral drug overdose. It aimed to define the patient characteristics and the types of drugs ingested, to evaluate the compliance with the department's protocols and to review the admission policy and to measure outcome.

\section{METHODS}

All patients who presented with a known or suspected oral drug overdose between 1st February 1989 and 31st July 1989 were studied. The details of these patients as explained below were collected on presentation by the attending medical officer. 
At one time seen, details of name age and sex were recorded together with the type of drugs ingested, and the time at which they were taken. The type of drug taken was determined from the history given by the patient or a witness, together with any circumstantial evidence. In several cases it was only when the patient had recovered and was awaiting discharge that this information was obtained. It was also noted whether or not the drug ingestion included alcohol. The clinical details recorded at presentation included pulse rate, blood pressure, respiratory rate and Glasgow Coma Scale Score and the presence or absence of airway protective reflexes. The only drug toxicology levels measured routinely at presentation were paracetamol and ethanol. Any method used to prevent further absorption of an ingested drug was documented together with any specific therapy given and the subsequent management of the patient.

The Accident and Emergency department of the Royal Brisbane Hospital does not recommend routine orogastric lavage except in comatose and already intubated patients. Orogastric lavage in any patient who was not already intubated for another reason, was only considered for potentially dangerous ingestions of certain drugs as listed in Figure 1. If orogastric lavage was undertaken in a conscious patient the airway was protected with an endotracheal tube and the patient was paralysed and ventilated during the procedure. The technique followed during orogastric lavage was as described by Matthew \& Lawson (1979). A large bore tube was placed in the stomach and $200 \mathrm{ml}$ alloquots of tepid tap water were poured into and drained out of the stomach at intervals until the return fluid was clear. Activated charcoal was given orally or via a nasogastric tube to patients who were conscious on arrival and had no specific indication for orogastric lavage. The dose of activated charcoal given was $50 \mathrm{~g}$ of medicinal charcoal BPC. (Norit ' $\mathrm{C}$ ' Extra的 made up as a slurry in $150 \mathrm{mls}$ of $70 \%$ sorbitol and $150 \mathrm{mls}$ of water. Subsequent doses were all $50 \mathrm{~g}$ and were given to patients who it was thought would benefit from repeat doses as for example in theophylline overdose. For patients with a reduced level of consciousness the recommended management was as shown in Figure 1.

All patients, except those discharged immediately, were admitted to the Accident and Emergency observaton unit unless there was a specific reason requiring admission to a higher dependency unit (Intensive Care or Coronary Care) or the patient was expected to require observations for longer than $24 \mathrm{~h}$. The Accident and Emergency observation unit has 22 beds and is supervized by Consultant and Registrar staff in Accident and Emergency Medicine. It also has a separate acute Psychiatric team, led by a Registrar and including a Social Worker in Pyschiatry available $16 \mathrm{~h}$ per day for the assessment of psychiatric emergencies but who's work also covers the assessment of the overdose patient.

\section{RESULTS}

A total of 289 patients presented on 323 occasions to the Accident and Emergency Department during the period from 1st February to 31 st July $1989.9 \%$ attended on more than one occasion. 


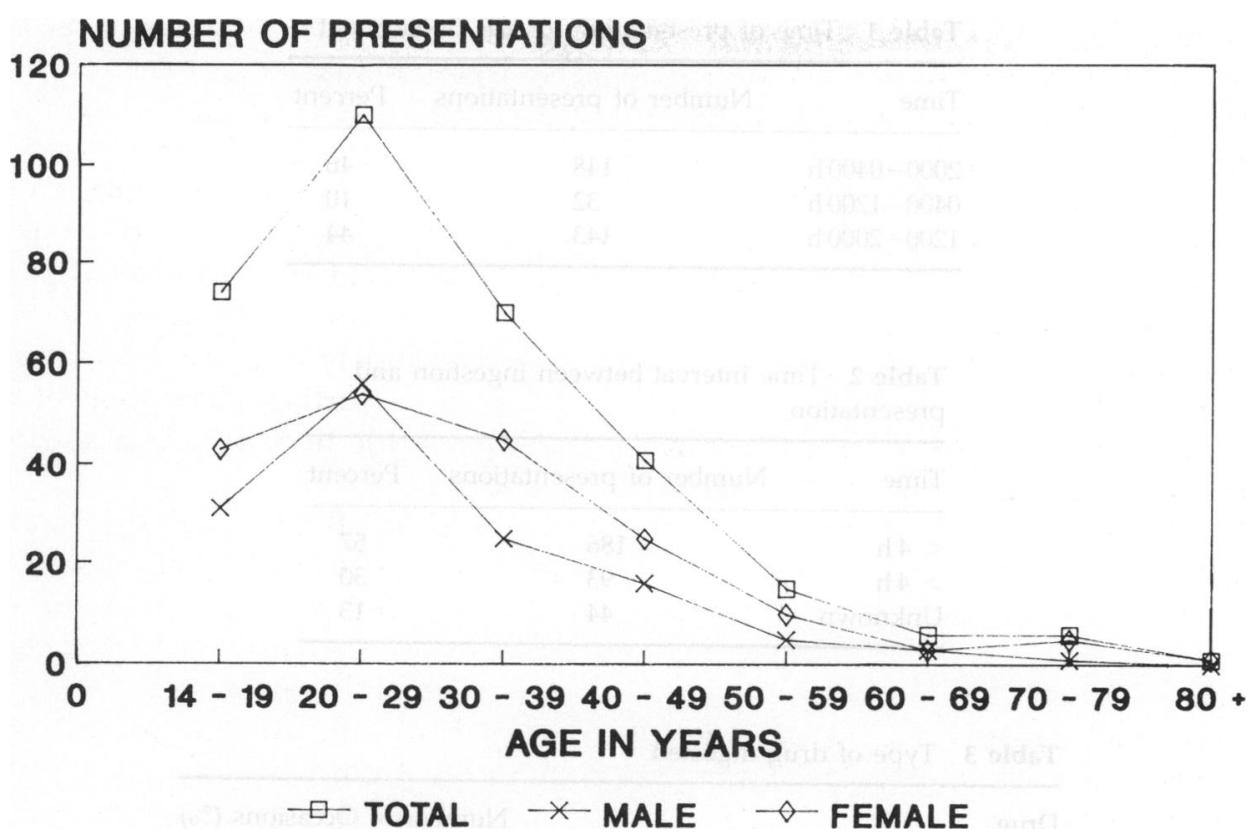

Fig. 2 Age distribution by sex of patients.

The age and sex distribution of patients is shown in Figure 2. A total of $90 \%$ of patients presented between $1200 \mathrm{~h}$ and $0400 \mathrm{~h}$ (Table 1) and $57 \%$ within 4 hours of ingestion (Table 2).

Table 3 shows the most common types of drugs taken. The benzodiazepines, ingested on their own or in combination was by far the most common group of drugs taken. The majority of patients $(61 \%)$ took only one drug. A total of $26 \%$ took two drugs and $13 \%$ took three or more drugs in combination. In $13 \%$ of patient presentations vomiting was known to have occurred prior to presentation. Alcohol was taken in association with an oral drug ingestion in $47 \%$ of male presentations and $30 \%$ of female presentations.

Paracetamol levels were recorded in 307 patient presentations out of the total of 323 and no treatable level of paracetamol was found in any patient who was not already suspected of taking paracetamol. The disposal of patients is shown in Figure 3 . The majority of patients $(76 \%)$ were managed totally in the Accident and Emergency observation unit and $23 \%$ were formally admitted to hospital inpatient beds.

Thirteen patients $(4 \%)$ underwent orogastric lavage -10 of these had an existing indication for endotracheal intubation and were lavaged following this procedure. Of the 10, six had taken a tricyclic overdose and four a barbiturate overdose. In the remaining three, orogastric lavage was undertaken in patients conscious at presentation, two had taken a tricyclic antidepressant overdose and one B-blocker overdose.

Thirteen patients $(4 \%)$ were given syrup of ipecac. Of these, one patient had 
12 D. W. Hodgkinson et al.

Table 1 Time of presentation to A/E Department

\begin{tabular}{lcc}
\hline Time & Number of presentations & Percent \\
\hline $2000-0400 \mathrm{~h}$ & 148 & 46 \\
$0400-1200 \mathrm{~h}$ & 32 & 10 \\
$1200-2000 \mathrm{~h}$ & 143 & 44 \\
\hline
\end{tabular}

Table 2 Time interval between ingestion and presentation

\begin{tabular}{lcc}
\hline Time & Number of presentations & Percent \\
\hline$<4 \mathrm{~h}$ & 186 & 57 \\
$>4 \mathrm{~h}$ & 93 & 30 \\
Unknown & 44 & 13 \\
\hline
\end{tabular}

Table 3 Type of drug ingested

\begin{tabular}{llc}
\hline Drug & & Number of Occasions (\%) \\
\hline Benzodiazepine & & $166(51 \cdot 4)$ \\
Antidepressant & (Tricyclic & $61(18 \cdot 8)$ \\
& (MAOI & 1 \\
Analgaesic & (Paracetamol & $48(14 \cdot 8)$ \\
& (Salicylate & $12(3 \cdot 7)$ \\
Antipsychotic & (NSAID & $14(4 \cdot 3)$ \\
Hypnotic/Sedative & (Chloral Hydrate & $24(7 \cdot 4)$ \\
& (Barbiturate & $8(2 \cdot 4)$ \\
Anticonvulsant & & $12(3 \cdot 7)$ \\
Antihistamine & & $12(3 \cdot 7)$ \\
Theophylline & & $14(4 \cdot 8)$ \\
Others & & $9(2 \cdot 7)$ \\
\hline
\end{tabular}

These are absolute numbers and include multiple drug ingestions by the same patient.

taken a sedative drug (tricyclic antidepressant) and presented within $20 \mathrm{~min}$ of $\stackrel{9}{3}$ taking the tablets. The remainder all presented within $4 \mathrm{~h}$, eight of these within $\frac{7}{0}$ $1 \mathrm{~h}$, and had taken a variety of non sedative drugs. All vomited within $30 \mathrm{~min}$ of being given $30 \mathrm{mls}$ of syrup of ipecac with water except one who needed two doses before vomiting.

In 196 patient presentations activated charcoal was given, 26 via a nasogastric tube and 170 orally. Of the patients given activated charcoal orally, $15 \%$ did not tolerate the suspension and refused to finish the $50 \mathrm{~g}$ dose. A further $21 \%$ of patients vomited within $4 \mathrm{~h}$ of being given activated charcoal. A total of $35 \%$ of $\stackrel{\oplus}{\stackrel{\oplus}{?}}$ patients who were given the activated charcoal and sorbitol slurry developed 


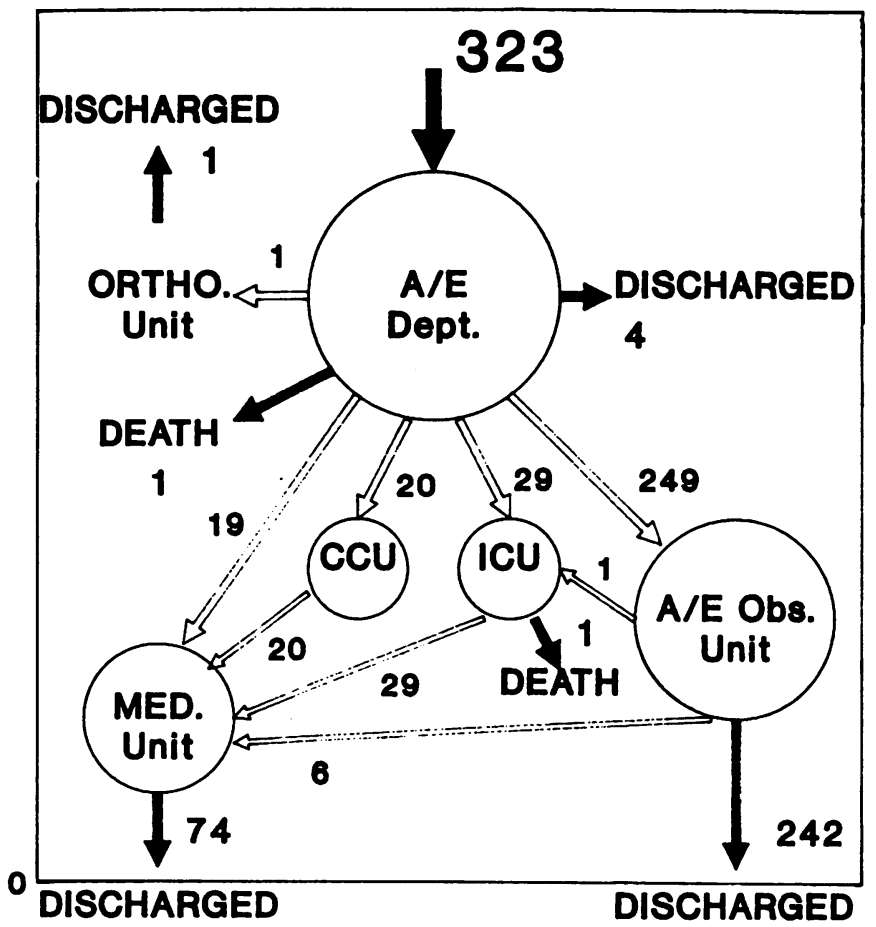

Fig. 3 Disposal of patients from A/E Department.

diarrhoea during admission. There were no other specific complications which may have been related to the use of activated charcoal and sorbitol.

Table 4 shows the total number of complications. There were five patients who aspirated gastric contents and all were admitted to the Intensive Care unit. One was a patient who deteriorated in the Accident and Emergency observation unit and who was thought to have aspirated during an unseccessful attempt at a difficult intubation by an experienced anaesthetist. Another was a patient who had signs of aspiration pneumonia on presentation. The other three were patients with Glasgow Coma Scale Scores of three at presentation, who were intubated and ventilated without delay and who subsequently developed signs of aspiration. The criteria used for diagnosing aspiration were based on clinical signs and radiological changes. The drugs associated with the aspiration of gastric contents

Table 4 Complications in all patients

\begin{tabular}{lc}
\hline Complication & Number of patients \\
\hline Death & 2 \\
Aspiration & 5 \\
Persistent vomiting & 1 \\
Abdominal pain of uncertain origin & 1 \\
Urinary tract infection & 1 \\
\hline
\end{tabular}


were tricyclic antidepressants, benzodiazepines, barbiturates and carbamazepine.

There were two deaths. One was a 66-year-old female who had taken a tricyclic antidepressant and chlorpromazine. She had an out-of-hospital cardiac arrest, was resuscitated in the Accident and Emergency Department but died $48 \mathrm{~h}$ later in the Intensive Care unit. The other was a 22-year-old male who had reportedly taken $3.5 \mathrm{~g}$ of a tricyclic antidepressant. On presentation he had a Glasgow Coma Scale Score of three, a pulse rate of 140 beats per min, a systolic blood pressure of $90 \mathrm{mmHg}$ and an arterial $\mathrm{pH}$ of $7 \cdot 05$. He had a cardiac arrest during intubation, was resuscitated and then had a further cardiac arrest $20 \mathrm{~min}$ later from which he could not be resuscitated. His serum dothiepin level taken at presentation was $1110 \mathrm{mcg} / \mathrm{L}$.

\section{DISCUSSION}

Oral drug overdose patients represent an important group of patients presenting to an Accident and Emergency Department. At the Royal Brisbane Hospital these patients comprise $1.4 \%$ of all presentations to the resuscitation and acute areas of the Accident and Emergency Department. The patients in this Australian study are similar to those presenting to Accident and Emergency Departments in the U.K. (Willox, 1985; Adams, 1986). The in-hospital mortality in our study was $0.3 \%$ which is comparable with other published work (Adams, 1986).

Of patient presentations, $77 \%$ were initially managed in the Accident and Emergency observation unit, and of these, $97 \%$ were discharged home from that unit without complication, indicating a high accuracy of triage. The death register for the Brisbane area was checked to ensure that none of these patients had subsequently died. Of the seven patients who required admission to hospital from the Accident and Emergency observation unit all were discharged well, although one patient had suffered an aspiration pneumonia. In our study oral drug overdose patients represented only $2 \cdot 1 \%$ of the total number of acute medical admissions to the Royal Brisbane Hospital. This figure was lower than reported in other studies (Matthew \& Lawson, 1979; Adams, 1986) and can be explained by the majority of patients in this study being managed in the Accident and Emergency observation unit. The management of overdose patients in a $24 \mathrm{~h}$ observation unit may be more efficient and cost effective than admission to hospital and would appear to be equally safe. This may have economic and bed utilisation implications.

Methods of preventing further drug absorption are best employed at an early stage in patient management. Orogastric lavage was utilized in $4 \%$ of patient presentations. This is in direct contrast to other studies where up to $80 \%$ of patients underwent orogastric lavage (Adams, 1986; Willox, 1985). This conservative approach to the use of orogastric lavage is supported by other authors (Proudfoot, 1984; Kulig, 1985) and did not adversely affect morbidity and mortality when compared with other methods (Adams, 1986).

The use of syrup of ipecac in adults is not recommended by the Royal Brisbane Hospital Accident and Emergency Department because it is no better than activated 
charcoal alone at preventing drug absorption (Neuvonen et al., 1983; Vale et al., 1986). In addition syrup of ipecac has the added problem of rare but significant side effects and complications such as cardiac and central nervous system toxicity (Vale et al., 1986; Wheeler-Usher et al., 1986). In this study $4 \%$ of patients received syrup of ipecac inspite of it not being recommended. This can be explained by a few doctors not following a recommended protocol. These patients did not suffer any complications inspite of one patient who had taken a large dose of a sedative drug being given syrup of ipecac. Kulig et al. (1985) examined the question of whether gastric emptying procedures are useful and found that syrup of ipecac did not alter the clinical course of poisoned patients who were alert and cooperative on presentation to hospital. These authors also noted that in the patients who were comatose, intubated or uncooperative, a clinically significant improvement occurred if gastric lavage followed by gastric instilled activated charcoal was undertaken within $1 \mathrm{~h}$ of ingestion. After $1 \mathrm{~h}$ there was no demonstrable difference between this group and those who received only activated charcoal.

A number of studies have examined and compared the efficacy of syrup of ipecac, gastric lavage and activated charcoal in normal volunteers (Neuvonen et al., 1983; Curtis et al., 1984; Tenebein et al., 1987; Albertson, 1989). These studies support the use of activated charcoal. The usefulness of activated charcoal in the treatment of acute drug ingestion has been reviewed in detail by several authors (Park et al., 1986; Derlet \& Albertson, 1986). Certain substances are not absorbed by activated charcoal for example ethanol, hydrocarbons and iron compounds. It is the preferred method of preventing further absorption of an orally ingested drug in overdose patients presenting to the Royal Brisbane Hospital. There were no serious complications related to the use of activated charcoal in combination with sorbitol. A high percentage of patients $(21 \%)$ vomited after being given the charcoal and sorbitol slurry. This value is higher than previously reported (Minocha et al., 1986) and was probably due to the sorbitol component of the slurry.

In the present climate of increasing accountability, systematic and critical analysis of the quality of medical care is becoming a necessity. The implications are not only clinical but also economic and should ensure the optimum quality of service within the resources available. We would recommend that Accident and Emergency departments dealing with oral drug overdose in adult patients use an algorithm similar to that proposed in Fig. 1 which is based on the exclusive use of activated charcoal together with sorbitol in the majority of patients.

\section{REFERENCES}

Adams R. H. M. (1986) An Accident and Emergency Department's view of self poisoning: a retrospective study from the United Norwich Hospitals 1978-1982. Human Toxicology 5, 5-10.

Albertson T. E., Derlet R. W., Foulke G. E. et al. (1989) Superiority of Activated charcoal alone compared with Ipecac and Activated charcoal in the treatment of acute toxic ingestions. Annals of Emergency Medicine 18, 101-4.

Curtis R. A., Barone J. and Giacona N. (1984) Efficacy of ipecac and Activated charcoal/cathartic prevention of salicylate absorption in a simulated overdose. Archives of Internal Medicine 144, 48-52. 
Derlet R. W. \& Albertson T. E. (1986) Activated charcoal: past, present and future. Western Journal of Medicine 145, 493-6.

Kulig K., Bar-Or D., Cantrill S. V. et al. (1985) Management of acutely poisoned patients without gastric emptying. Annals of Emergency Medicine 14, 562-7.

Matthew H. and Lawson A. H. (1979) Treatment of common acute poisoning. Ch. 5 Churchill Livingstone, Edinburgh pp. 29-43.

Melman K. L. and Morelli H. F. (1978) Clinical pharmacology - second edition Macmillan, New York.

Minocha A. et al. (1986) Superior efficacy of sorbitol cathartics in poisoned patients. AAPCC/AACT/ AMBT/CAPCC Annual Scientific meeting. Santa Fe, New Mexico. Abstract 112.

Neuvonen P. J., Vartiainen M., Tokola O. et al. (1983) Comparison of Activated charcoal and ipecac syrup in prevention of drug absorption. European Journal of Clinical Pharmacology 24, 557-62.

Nielson M. \& Henry J. (1984) ABC of poisoning. British Medical Journal 289, 614-618.

Park G. D., Spector R., Goldberg M. J. et al. (1986) Expanded role of charcoal therapy in the poisoned and overdosed patient. Archives of Internal Medicine 146, 969-73.

Proudfoot A. T. (1984) Abandon gastric lavage in the accident and emergency department. Archives of Emergency Medicine 2, 65-71.

Thompson A. M., Robins J. B. \& Prescott L. F. (1987) Changes in cardiorespiratory function during gastric lavage for drug overdose. Human Toxicology 6, 215-8.

Tenebein M., Cohen S. \& Sitar D. S. (1987) Efficacy of ipecac-induced emesis, orogastric lavage, and activated charcoal for acute drug absorption. Annals of Emergency Medicine 16, 838-41.

Vale J. A., Meredith T. J. \& Proudfoot A. T. (1986) Syrup of ipecac: is it really useful. British Medical Journal 293, 1341.

Wheeler-Usher D. H., Wanke L. A., Bayer M. J. et al. (1986) Gastric emptying risk versus benefit in the treatment of acute poisoning. Medical Toxicology 1, 142-53.

Willox D. G. (1985) Self poisoning: A review of patients seen in the Victoria Royal Infirmary Glasgow. Scottish Medical Journal 30, 220-224. 